\title{
Luminescent Dinuclear Lanthanide Complexes of 5-Me-HXTA
}

Louise S. Natrajan, ${ }^{a}$ Phillipa L. Timmins, ${ }^{b}$ Matthew Lunn, ${ }^{c}$ and Sarah .L Heath ${ }^{* i a}$

${ }^{a}$ Department of Chemistry, University of Manchester, Oxford Road, Manchester, M13 9PL. Fax: 0161 254598 ; Tel: 0161275 4696: sarah.l.heath@man.ac.uk, ${ }^{b}$ Department of Chemistry, University of York, Heslington, York, YO10 5DD, ${ }^{c}$ Department of Chemistry, University of Sheffield, Brook Hill, Sheffield, S3 $7 H F$

\section{Supplementary Information}

Figure S1. ${ }^{1} \mathrm{H}$ DOSY NMR spectrum of $\mathrm{Na}_{4} .5-\mathrm{Me}-\mathrm{HXTA}, \mathrm{D}_{2} \mathrm{O}, 295 \mathrm{~K}$

Novil

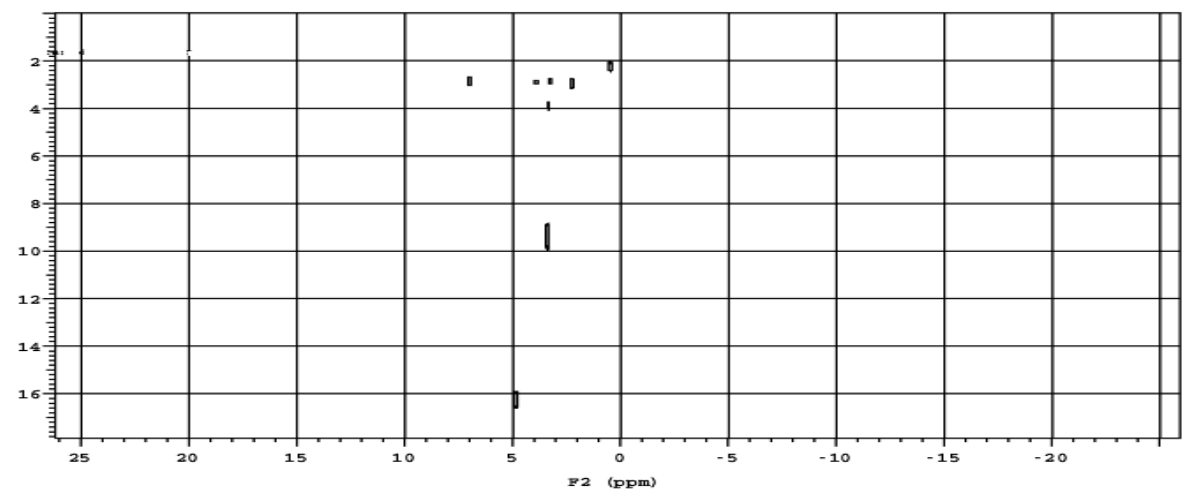

Figure S2. ${ }^{1} \mathrm{H}$ DOSY NMR spectrum of 1:1 L:M solutions of $\mathrm{Na}_{4} .5-\mathrm{Me}-\mathrm{HXTA}$ and $\mathrm{Eu}(\mathrm{OTf})_{3}, \mathrm{D}_{2} \mathrm{O}, 295 \mathrm{~K}$

de Maldedl

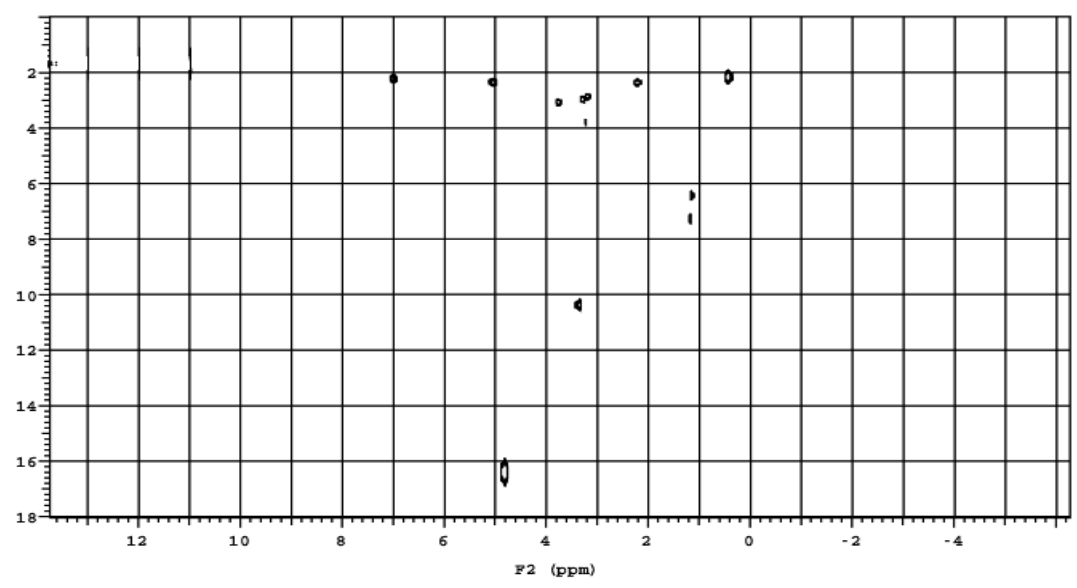


Figure S3. ${ }^{1} \mathrm{H}$ DOSY NMR spectrum of $\mathrm{Na}_{4} \cdot[\mathrm{Nd}(5-\mathrm{Me}-\mathrm{HXTA})]_{2}(\mathbf{1}), \mathrm{D}_{2} \mathrm{O}, 295 \mathrm{~K}$

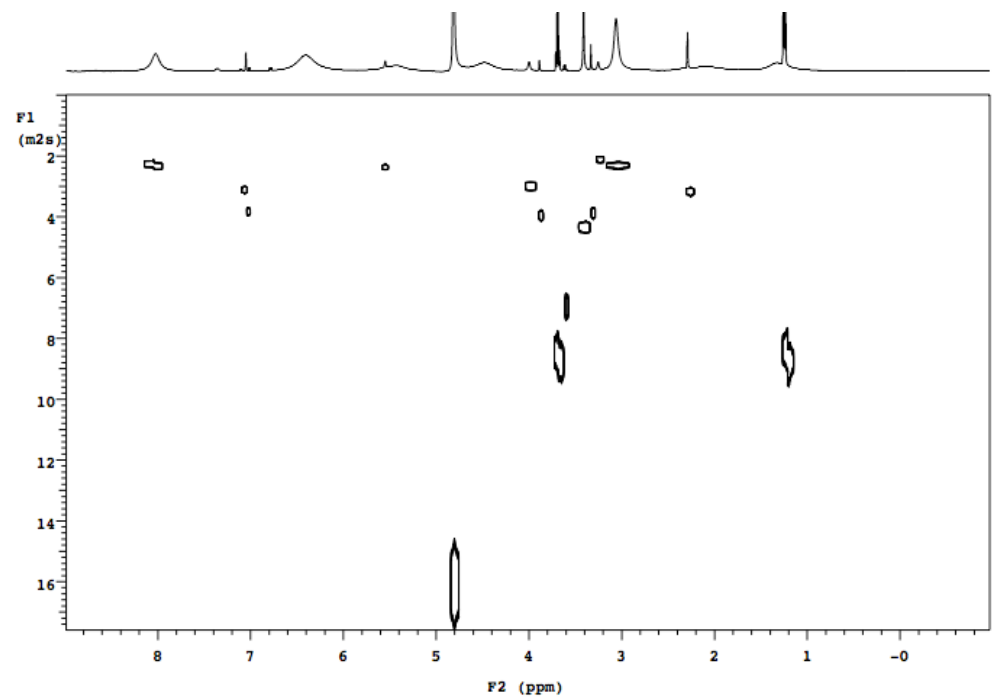

Figure S4. Electrospray mass spectrum (negative mode) of $\mathrm{Na}_{4} \cdot[\mathrm{Tb}(5-\mathrm{Me}-\mathrm{HXTA})]_{2}$ (4)

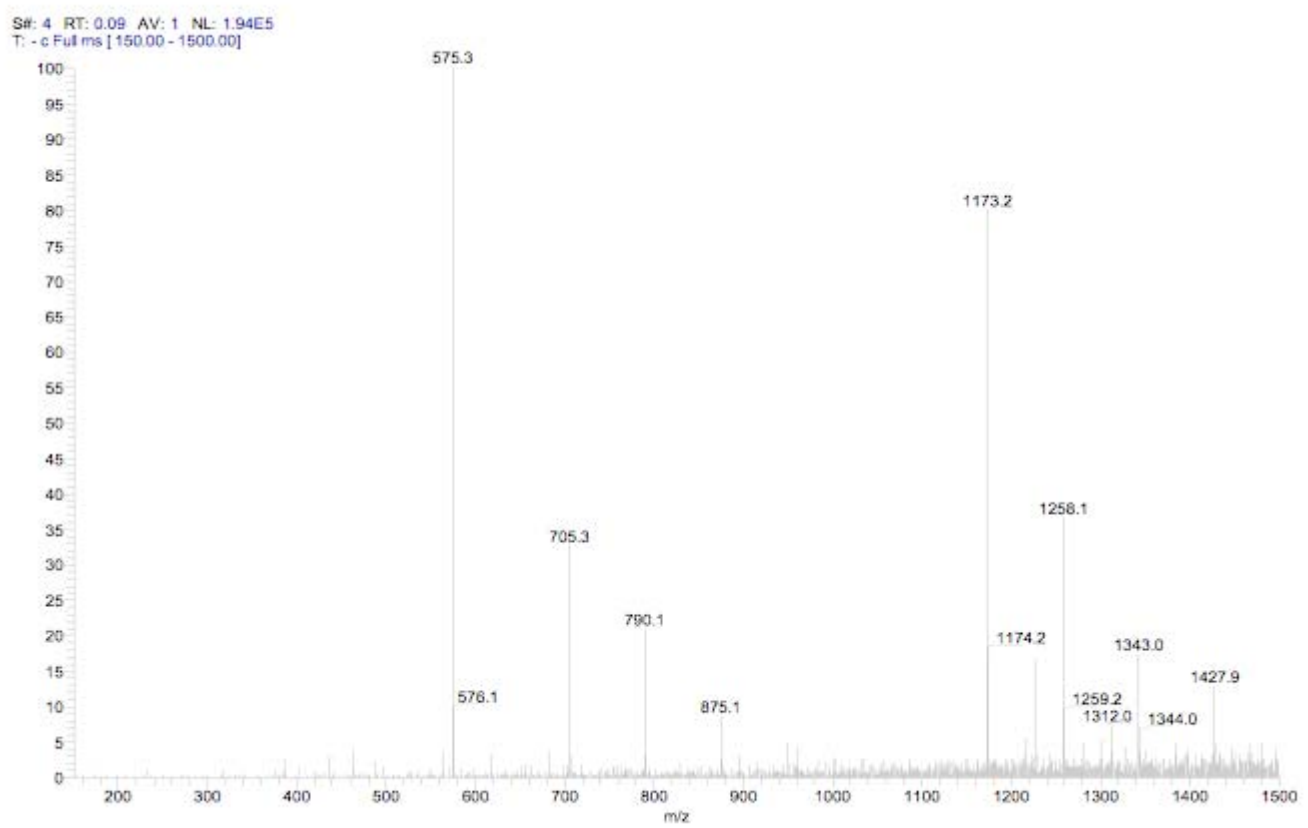


Figure S5. Fitted kinetic trace for $[\mathrm{Tb}(\text { HXTA })]_{2} \cdot \mathrm{Na}_{4}(\mathbf{4})$ in $\mathrm{D}_{2} \mathrm{O}\left({ }_{\text {exc }}=300 \mathrm{~nm}, \quad\right.$ em $=$ $545 \mathrm{~nm}, \quad$ н2О $=2.91 \mathrm{~ms}$ )

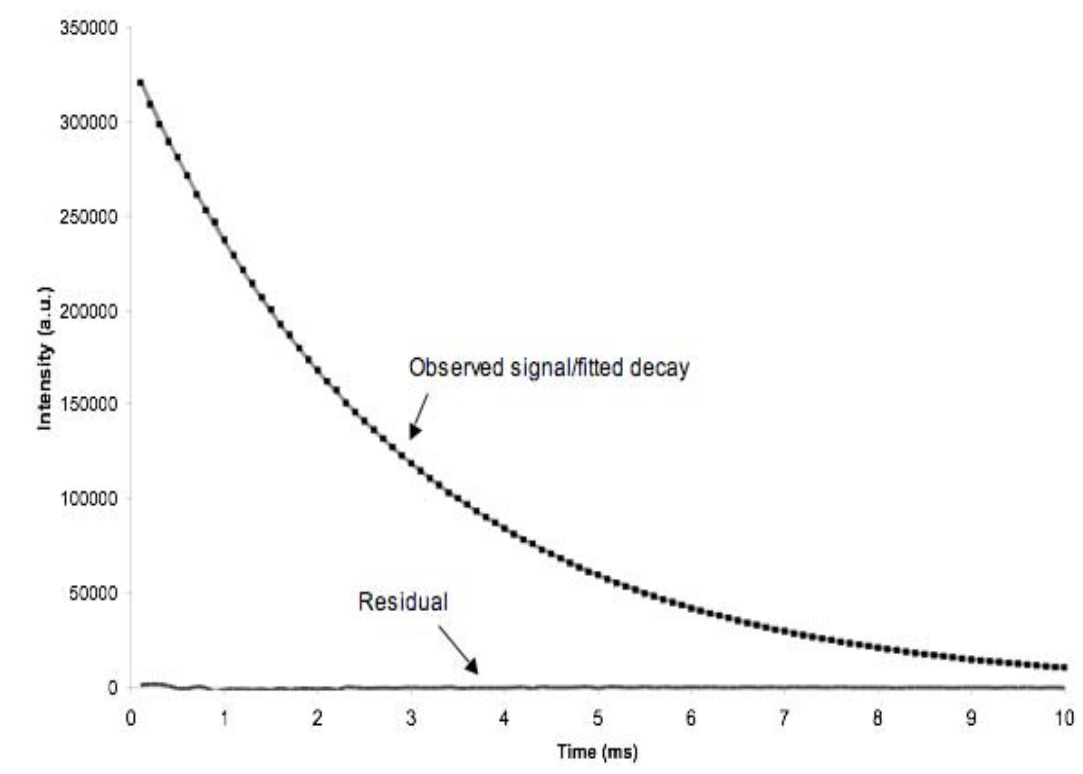

Figure S6. Steady state emission spectra of $\mathrm{Na}_{4} \cdot[\mathrm{Tb}(5-\mathrm{Me}-\mathrm{HXTA})]_{2}$ (4) at low concentrations $\left(\lambda_{\mathrm{exc}}=300 \mathrm{~nm}, \lambda_{\mathrm{em}}=545 \mathrm{~nm}, 10 \mathrm{~nm}\right.$ slits, gate time $=0.1 \mathrm{~ms}$, delay time $=0.1 \mathrm{~ms}$ ); the ${ }^{5} \mathrm{D}_{4} \rightarrow{ }^{7} \mathrm{~F}_{4}$ peak at $587 \mathrm{~nm}$ contains a contribution from a $2 \lambda$ artefact $(600 \mathrm{~nm})$

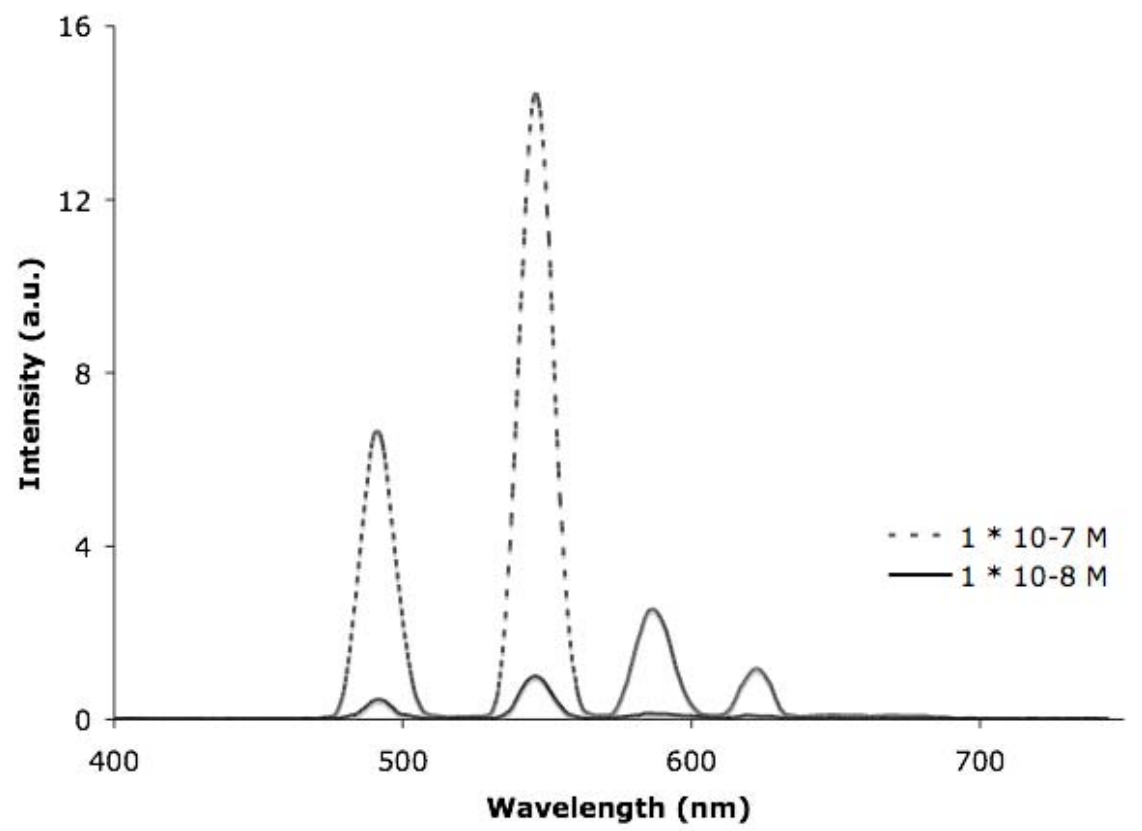




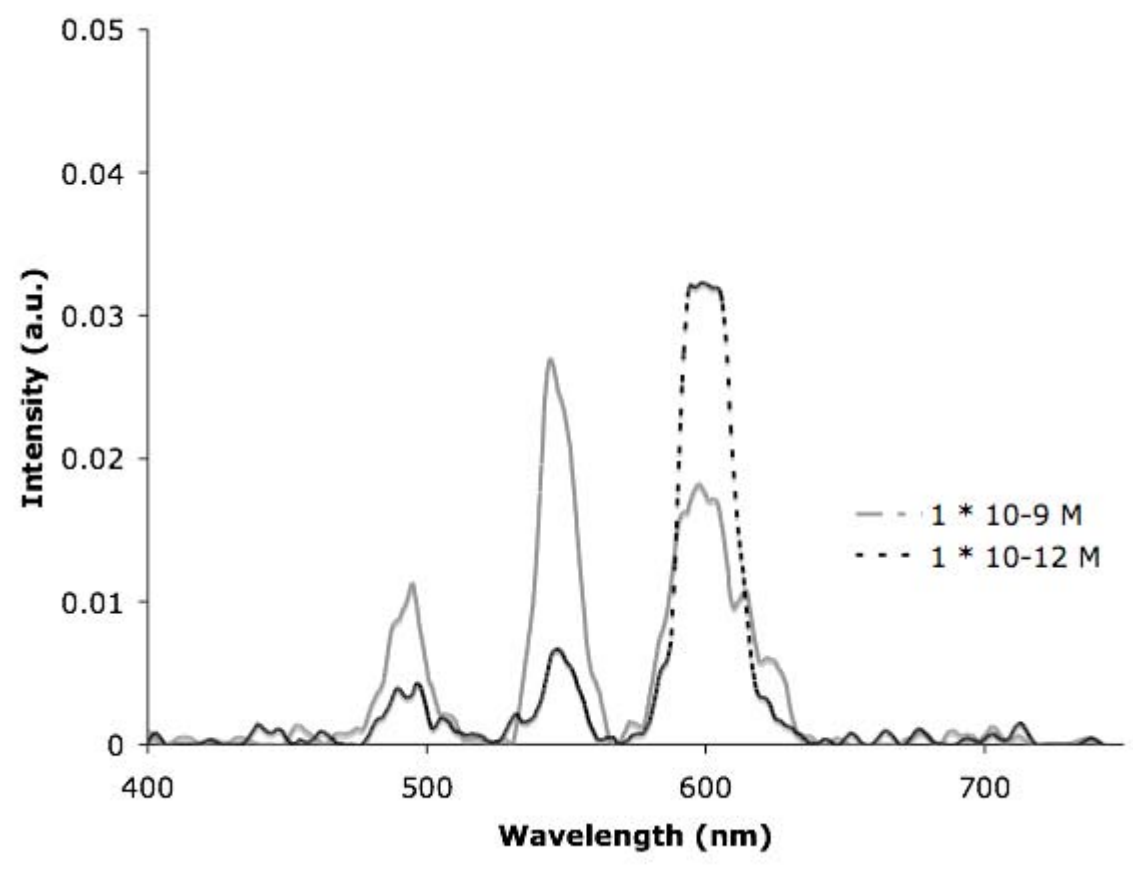

Figure S7. Absorption spectrum of $\mathrm{Na}_{4}$.[Eu(5-Me-HXTA) $]_{2}(3), \mathrm{H}_{2} \mathrm{O}, 298 \mathrm{~K}$

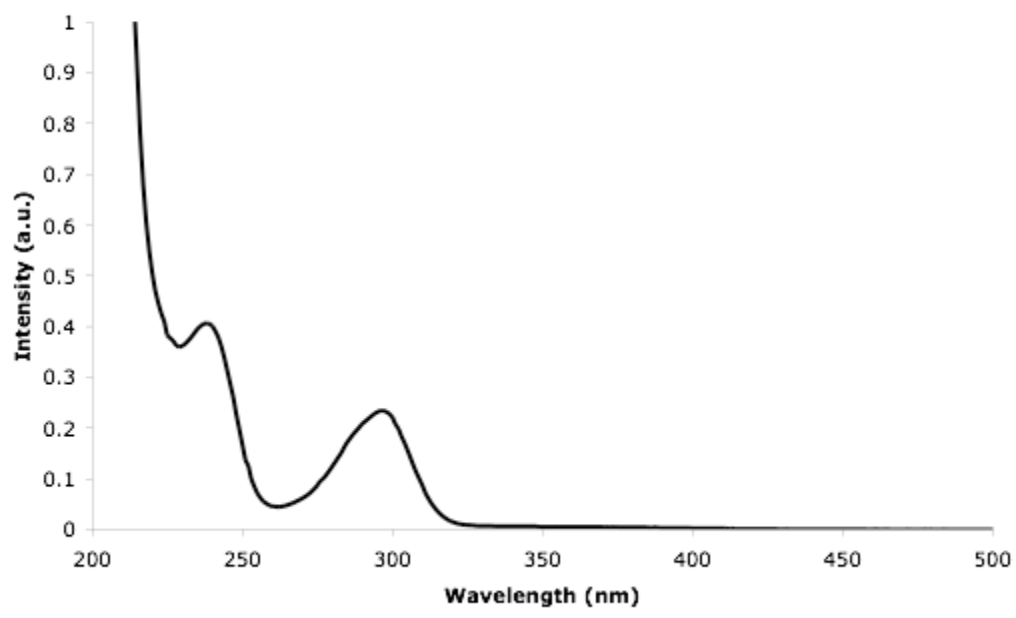


Figure S8. Absorption spectrum of $\mathrm{Na}_{4}$.[Eu(5-Me-HXTA) $]_{2}$ (3) showing the LMCT absorption band at $\lambda_{\max }=362 \mathrm{~nm}, \mathrm{H}_{2} \mathrm{O}, 298 \mathrm{~K}$

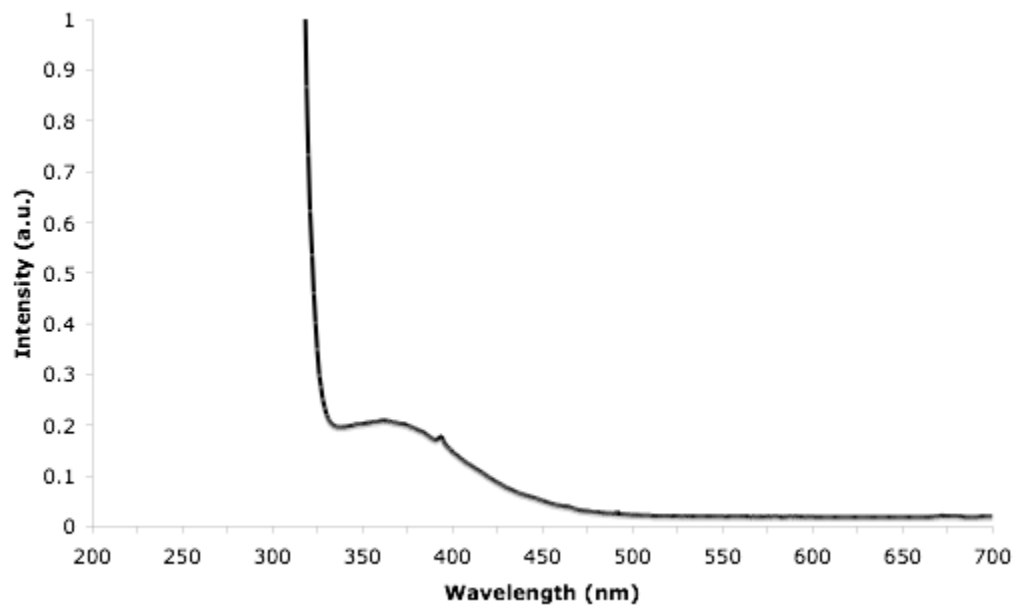

Figure S9. Fitted time resolved decay profile for $[\mathrm{Nd}(\text { HXTA })]_{2} \cdot \mathrm{Na}_{4}(\mathbf{1})$ in $\mathrm{D}_{2} \mathrm{O}($ exc $=$ $337 \mathrm{~nm}, \quad$ em $\left.=1055 \mathrm{~nm}, \quad{ }_{\mathrm{D} 2 \mathrm{O}}=0.32 \mu \mathrm{s}\right)$

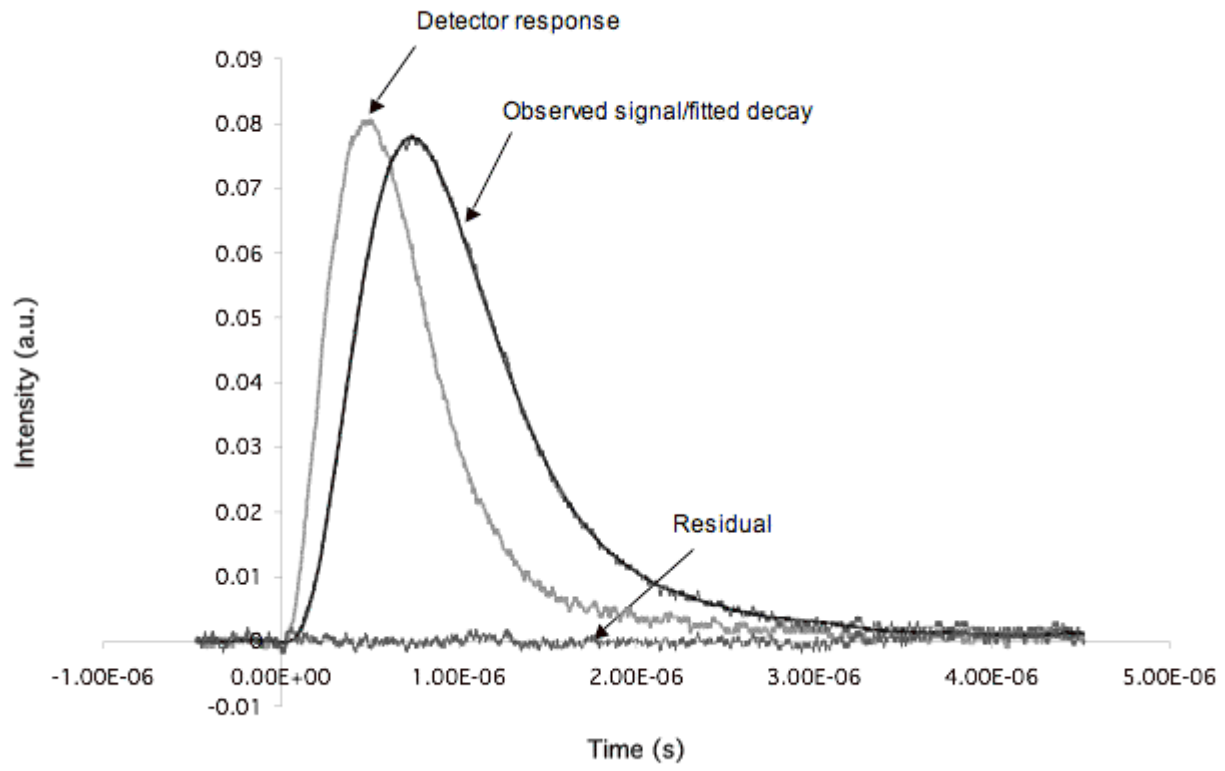


Figure S10. Fitted time resolved decay profile for $[\mathrm{Nd}(\mathbf{H X T A})]_{2} \cdot \mathrm{Na}_{4}(\mathbf{1})$ in $\mathrm{H}_{2} \mathrm{O}$ ( exc $\left.=337 \mathrm{~nm}, \quad \mathrm{em}=1055 \mathrm{~nm}, \quad{ }_{\mathrm{D} 2 \mathrm{O}}=0.10 \mu \mathrm{s}\right)$

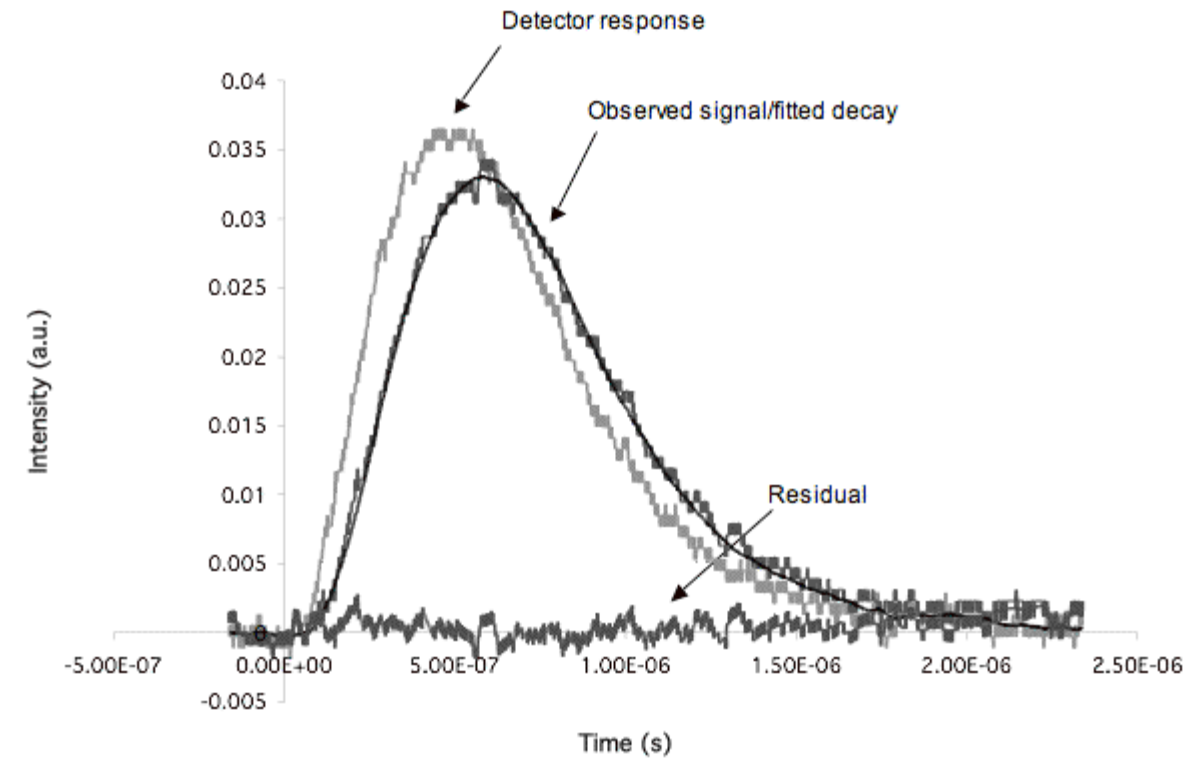

Figure S11. Time resolved emission spectrum of $[\mathrm{Yb}(\text { HXTA })]_{2} \cdot \mathrm{Na}_{4}(\mathbf{8})$ in $\mathrm{D}_{2} \mathrm{O}($ exc $=337 \mathrm{~nm}, \quad \mathrm{em}=980 \mathrm{~nm}, \quad$ D2O $=7.51 \mu \mathrm{s})$

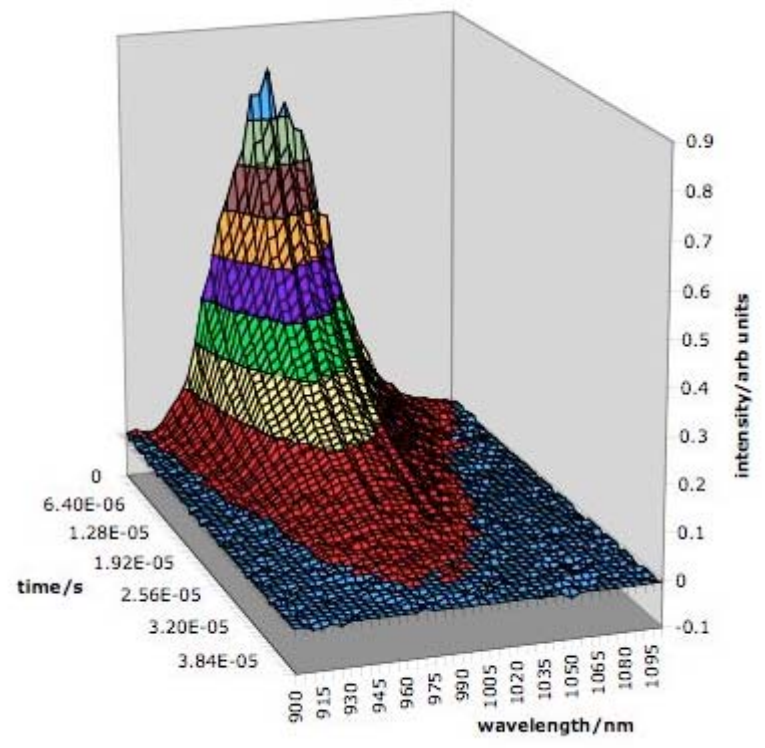


Figure S12. Fitted time resolved decay profile for $[\mathrm{Yb}(\mathbf{H X T A})]_{2} \cdot \mathrm{Na}_{4}(8)$ in $\mathrm{H}_{2} \mathrm{O}$ ( exc $=337 \mathrm{~nm}, \quad \mathrm{em}=980 \mathrm{~nm}, \quad$ D2O $=2.14 \mu \mathrm{s})$

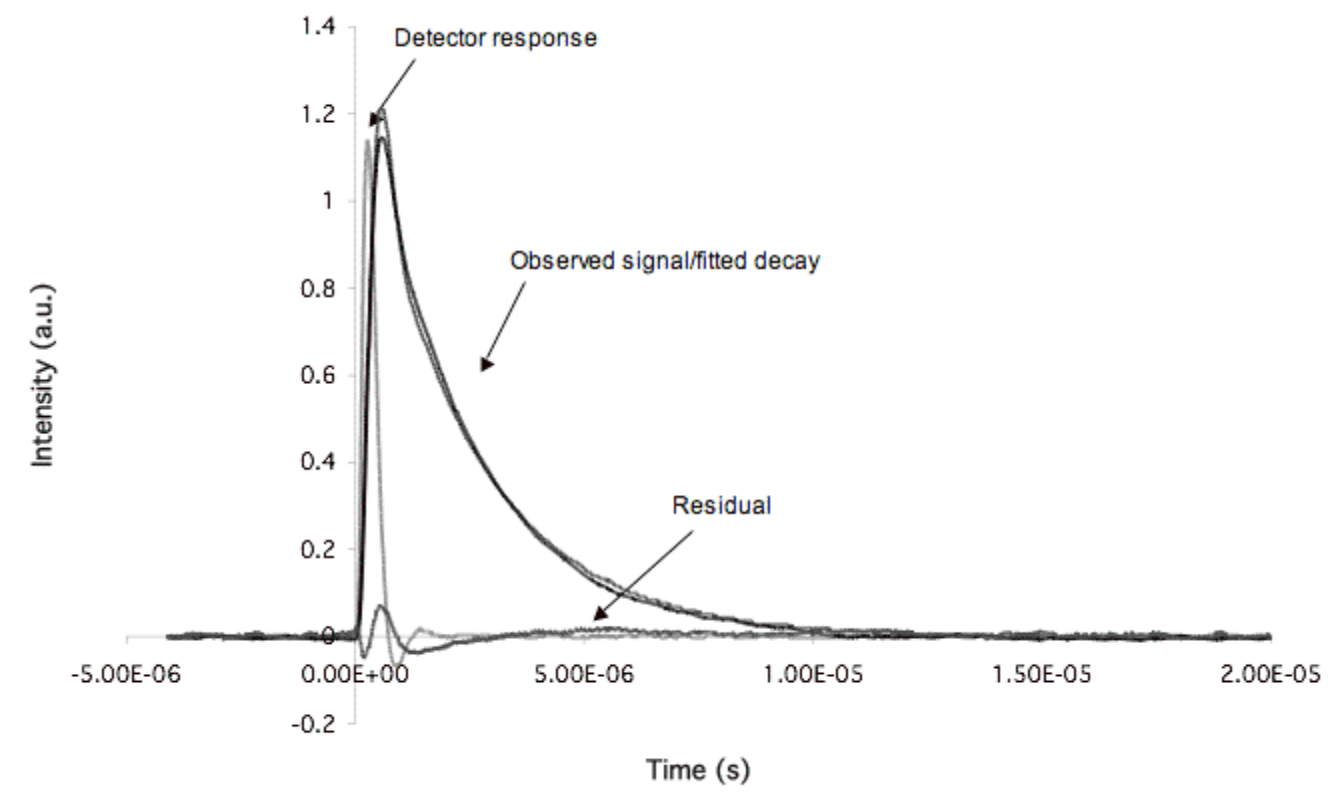

